Reprod. Nutr. Dévelop., 1986, 26 (2 B), 637-642.

\title{
La thermogenèse induite par l'alimentation chez le rat Zucker âgé de 2 et 7 jours
}

Eléonore PLANCHE, M. JOLIFF

Laboratoire sur la Physiopathologie de la Nutrition. INSERM U.177, Institut Biomédical des Cordeliers, 15, rue de l'Ecole de Médecine, 75006 Paris.

Summary. Diet-induced thermogenesis in 2 and 7-day old Zucker rats.

Gas exchanges were measured at the thermoneutral temperature of $35^{\circ} \mathrm{C}$ on " fasted » $(3 \mathrm{~h} 30 \mathrm{~min}$ ) and then « refed $»(75 \mathrm{~min}) \mathrm{fa} / \mathrm{fa}$ and $\mathrm{Fa} / \mathrm{fa}$ rats aged 2 and 7 days. The $\mathrm{CO}_{2}$ production, $\mathrm{O}_{2}$ consumption and respiratory quotient increased significantly after refeeding in all pups. The percentage of increase in the gas exchanges was similar in both genotypes at 2 days. At 7 days, the percentage of increase was significantly higher in $\mathrm{Fa} / \mathrm{fa}$ than in $\mathrm{fa} / \mathrm{fa}$ pups. This demonstrated a defect in diet-induced thermogenesis in 7-day old pups.

However, since this defect (unlike non-shivering thermogenesis) was absent in 2-day old pups, it is concluded that it is probably not a primary factor but rather a consequence of the obesity already present in fa/fa pups at 7 days of age.

\section{Introduction.}

Le rôle étiologique d'un déficit de la thermogenèse dans l'installation et le développement de plusieurs types d'obésités génétiques a été confirmé par des études sur les animaux avant le sevrage, notamment sur le rat Zucker obèse homozygote pour le gène fa (Godbole et al., 1978 ; Kaplan, 1979 ; Planche et al., 1983 ; Schmidt et al., 1984 ; Planche et Joliff, 1985).

La thermogenèse, dont le site principal est le tissu adipeux brun (Foster et Frydman, 1979), peut être induite par deux types de stimuli, le froid ou la prise alimentaire. Chez le $\mathrm{fa} / \mathrm{fa}$ adulte le rôle respectif d'une anomalie de l'un ou l'autre de ces types de thermogenèse dans le développement de l'obésité est controversé. Certains auteurs (Trayhurn et al., 1976 ; Schmidt et al., 1984 ; Stern et al., 1984) observent chez le $\mathrm{fa} / \mathrm{fa}$ une diminution de la thermogenèse induite par le froid ; d'autres (Rothwell et al., 1981 ; Triandafillou et Himms-Hagen, 1983 ; Holt et al., 1983) attribuent son déficit énergétique uniquement à une anomalie de la thermogenèse induite par l'alimentation. Chez le fa/fa avant le sevrage, la part respective de ces deux types de thermogenèse dans le déficit de la dépense énergétique n'a pas encore été étudiée. Dans un précédent travail (Planche et Joliff, 
1985) nous avions montré qu'un déficit de la thermogenèse était présent chez le $\mathrm{fa} / \mathrm{fa}$ dès l'âge de 2 jours et qu'il semblait principalement dû à une anomalie de la thermogenèse induite par le froid puisqu'il était observé seulement lorsque la température ambiante était abaissée au-dessous de $35^{\circ} \mathrm{C}$, température de neutralité thermique (Conklin et Heggeness, 1971). A $35^{\circ} \mathrm{C}$, nous ne trouvions pas de différences entre les deux génotypes. Comme les animaux étaient étudiés à l'état nourri, nous avions émis I'hypothèse que, aux âges étudiés : 2,5 et 7 jours, la thermogenèse induite par l'alimentation était normale chez le fa/fa. Cependant, l'état nutritionnel réel des animaux et, en particulier, ie moment de la dernière prise alimentaire n'était pas connu. De possibles variations entre les ratons pouvaient avoir masqué des différences entre les $\mathrm{fa} / \mathrm{fa}$ et les $\mathrm{Fa} / \mathrm{fa}$.

Le travail actuel a été entrepris afin de tester l'effet de la réalimentation sur le niveau des dépenses énergétiques de rats Zucker âgés de 2 et 7 jours et de mettre éventuellement en évidence une anomalie de la thermogenèse induite par l'alimentation, pouvant jouer un rôle dans l'installation de l'obésité du rat fa/fa.

\section{Matériel et méthodes.}

Animaux. - Les animaux utilisés sont des rats Zucker obèses ( $\mathrm{fa} / \mathrm{fa}$ ) et non obèses $(\mathrm{Fa} / \mathrm{fa})$ âgés de 2 et 7 jours, nés du croisement d'un mâle obèse et d'une femelle hétérozygote. Les petits (10 par portée) et leur mère étaient conservés dans une animalerie éclairée de $7 \mathrm{~h}$ à $19 \mathrm{~h}$ et dont la température était $22 \pm 1{ }^{\circ} \mathrm{C}$. Après l'étude, les petits étaient rendus à leur mère et le génotype identifié à l'âge de 6 semaines.

Echanges gazeux. - Les mesures sont pratiquées à la température ambiante de $35^{\circ} \mathrm{C}$. La mesure conjointe de $\mathrm{I}^{\prime} \mathrm{O}_{2}$ consommé et du $\mathrm{CO}_{2}$ excrété est faite dans un appareil à circuit fermé, comme précédemment décrit (Planche et al., 1983). Tous les petits d'une même portée sont étudiés simultanément. Ils sont enlevés à la mère à $12 \mathrm{~h}$ et gardés pendant $3 \mathrm{~h}$ à $33^{\circ} \mathrm{C}$, température du nid (Planche et al., 1983). Ensuite, ils sont placés individuellement dans les chambres métaboliques. Après une période d'équilibration de $20 \mathrm{~min}$, les tubes contenant la soude destinée à récolter le $\mathrm{CO}_{2}$ expiré, sont changés et les échanges gazeux mesurés pendant $60 \mathrm{~min}$. Les petits (" à jeun » depuis $4 \mathrm{~h} 20 \mathrm{~min}$ ) sont alors pesés, marqués et rendus à leur mère pendant $75 \mathrm{~min}$. De nouveau, les petits " renourris " sont pesés et les échanges gazeux mesurés pendant $60 \mathrm{~min}$.

Le $\mathrm{CO}_{2}$ excrété, collecté dans $8 \mathrm{ml}$ de $\mathrm{NaOH} 1 \mathrm{~N}$, est mesuré par titrimétrie à l'aide d'un pH Stat (Radiometer, Copenhague). $L^{\prime} \mathrm{O}_{2}$ consommé est mesuré par lecture directe sur un réservoir gradué et corrigé pour la température et la pression atmosphérique (STPD). Les résultats sont exprimés en $\mathrm{ml}$ de $\mathrm{CO}_{2}$ et d $\mathrm{d}^{\prime} \mathrm{O}_{2}$ par $100 \mathrm{~g}$ de poids corporel.

Les différences entre les animaux "à jeun " et "renourris " étaient exprimées en pourcentage d'augmentation du $\mathrm{CO}_{2}$ excrété et de l' $\mathrm{O}_{2}$ consommé.

Statistiques. - Les comparaisons entre les deux génotypes sont faites en utilisant une analyse de variance à deux voies permettant d'éliminer les variations dues aux différences entre les portées (Snedecor et Cochran, 1967). 


\section{Résultats et discussion.}

Poids corporel et ingesta (tabl. 1). - Comme nous l'avons précédemment montré (Planche et al., 1983; Planche et Joliff, 1985), aux âges étudiés, le génotype n'a aucun effet sur le poids corporel des animaux.

\section{TABLEAU 1}

Poids des rats et ingesta.

\begin{tabular}{lcccccc}
\hline & \multicolumn{3}{c}{$2 \mathrm{~J}$} & \multicolumn{3}{c}{$7 \mathrm{~J}$} \\
\cline { 2 - 7 } & $\mathrm{fa} / \mathrm{fa}(24)$ & $\mathrm{Fa} / \mathrm{fa}(25)$ & $\mathrm{fa} / \mathrm{fa}(31)$ & $\mathrm{Fa} / \mathrm{fa}(27)$ \\
\hline Poids corporel $(\mathrm{g}) \ldots \ldots \ldots$ & $7,0 \pm 0,1$ & $7,1 \pm 0,1$ & $12,5 \pm 0,4$ & $12,3 \pm 0,2$ \\
\hline Ingesta $(\mathrm{mg}) \ldots \ldots \ldots \ldots$ & $193 \pm 23$ & $175 \pm 21$ & $267 \pm 38$ & $318 \pm 43$ \\
\hline
\end{tabular}

Les résultats sont exprimés en moyenne + S.E.M. pour le nombre d'animaux entre parenthèses.

Les ingesta étaient appréciés par la différence entre les poids des animaux avant et après réalimentation.

Nous n'avons pas observé non plus, de différence statistiquement sıgnificative entre les 2 génotypes, dans le niveau des ingesta, ce qui confirme, également, les observations d'autres groupes de chercheurs (Boulangé et al., 1979 ; Godbole et al., 1981).

Echanges gazeux (tabl. 2 et fig. 1). - Nos résultats montrent que, dans tous les groupes d'animaux, la réalimentation induit une augmentation de la thermogenèse, mise en évidence par l'élévation conjointe du niveau des échanges gazeux. Cette augmentation étant plus importante pour le $\mathrm{CO}_{2}$ que pour $\mathrm{I}^{\prime} \mathrm{O}_{2}$, il en résulte une élévation du quotient respiratoire (QR).

TABLE 2

Echanges gazeux et $Q$. R. a jeun et après réalimentation.

\begin{tabular}{|c|c|c|c|c|c|c|}
\hline \multirow{2}{*}{$\frac{\text { Age }}{\text { Génotype }}$} & \multicolumn{2}{|c|}{$\mathrm{CO}_{2}(\mathrm{ml} / \mathrm{h} / 100 \mathrm{~g})$} & \multicolumn{2}{|c|}{$\mathrm{O}_{2}(\mathrm{ml} / \mathrm{h} / 100 \mathrm{~g})$} & \multicolumn{2}{|c|}{ Q.R. } \\
\hline & A jeun & Renourris & A jeun & Renourris & A jeun & Renourris \\
\hline $\begin{array}{l}2 \text { Jours : } \\
\mathrm{fa} / \mathrm{fa}(24) \\
\mathrm{Fa} / \mathrm{fa}(25)\end{array}$ & $\begin{array}{l}109 \pm 4 \\
100 \pm 4\end{array}$ & $\begin{array}{l}126 \pm 4^{* *} \\
129 \pm 5^{* *}\end{array}$ & $\begin{array}{l}148 \pm 4 \\
150 \pm 5\end{array}$ & $\begin{array}{l}167 \pm 6^{* *} \\
170 \pm 7^{* *}\end{array}$ & $\begin{array}{l}0,73 \pm 0,01 \\
0,73 \pm 0,00\end{array}$ & $\begin{array}{l}0,76 \pm 0,00^{* *} \\
0,76 \pm 0,01^{* *}\end{array}$ \\
\hline $\begin{array}{l}7 \text { jours : } \\
\mathrm{fa} / \mathrm{fa}(31) \\
\mathrm{Fa} / \mathrm{fa}(27)\end{array}$ & $\begin{array}{l}114 \pm 1 \\
114 \pm 1\end{array}$ & $\begin{array}{l}125 \pm 1^{* *} \\
128 \pm 2^{* *}\end{array}$ & $\begin{array}{l}154 \pm 2 \\
157 \pm 2\end{array}$ & $\begin{array}{l}163 \pm 2^{* *} \\
171 \pm 3^{* *} \S\end{array}$ & $\begin{array}{l}0,74 \pm 0,00 \\
0,73 \pm 0,00\end{array}$ & $\begin{array}{l}0,77 \pm 0,01^{* *} \\
0,75 \pm 0,01^{*}\end{array}$ \\
\hline
\end{tabular}

Les échanges gazeux étaient mesurés chez des rats fa/fa et $\mathrm{Fa} / \mathrm{fa}$ " à jeûn 》 et « renourris " âgés de 2 et 7 jours. Les résultats sont les moyennes \pm SEM du nombre d'animaux entre parenthèses, les différences statistiquement significatives entre rats « à jeun " et renourris sont indiquées par * : $P<0,05,{ }^{*}: P<0,01$, et entre les 2 génotypes par $\S: P<0,01$. 
Cette augmentation du OR bien que statistiquement significative, était faible, probablement à cause du taux élevé des lipides dans le lait (Godbole et al., 1981), cependant elle confirme l'effet de la réalimentation sur le métabolisme énergétique des ratons. L'étude étant faite à la température de neutralité thermique, l'effet du froid sur le niveau des dépenses énergétiques pouvait être éliminé (Conklin et Heggeness, 1971 ; Planche et Joliff, 1985), et l'augmentation de ces dépenses pouvait être entièrement attribuée à la thermogenèse induite par l'alimentation. A l'âge de 2 jours les échanges gazeux augmentent d'une manière similaire dans les 2 génotypes (fig. 1). A 7 jours, le pourcentage d'augmentation est moindre qu'à 2 jours. Ceci peut être dû soit au fait qu'un " jeûne » de $4 \mathrm{~h} 20 \mathrm{~min}$ est plus long et en conséquence, la réalimentation plus effective à 2 jours qu'à 7 jours, ou que les ratons réagissent plus à des changements nutritionnels à 2 jours que plus tard, comme nous l'avions observé pour la réponse à I'abaissement de la température ambiante (Planche et Joliff, 1985). Cette diminution de la réponse à la réalimentation était significative chez le $f a / f a(2 \mathrm{~J}$ vs $7 \mathrm{~J}$ : $\mathrm{P}<0,01)$ mais non chez le $\mathrm{Fa} / \mathrm{fa}$, de sorte que la différence entre les 2 génotypes devenait statistiquement significative (fig. 1).

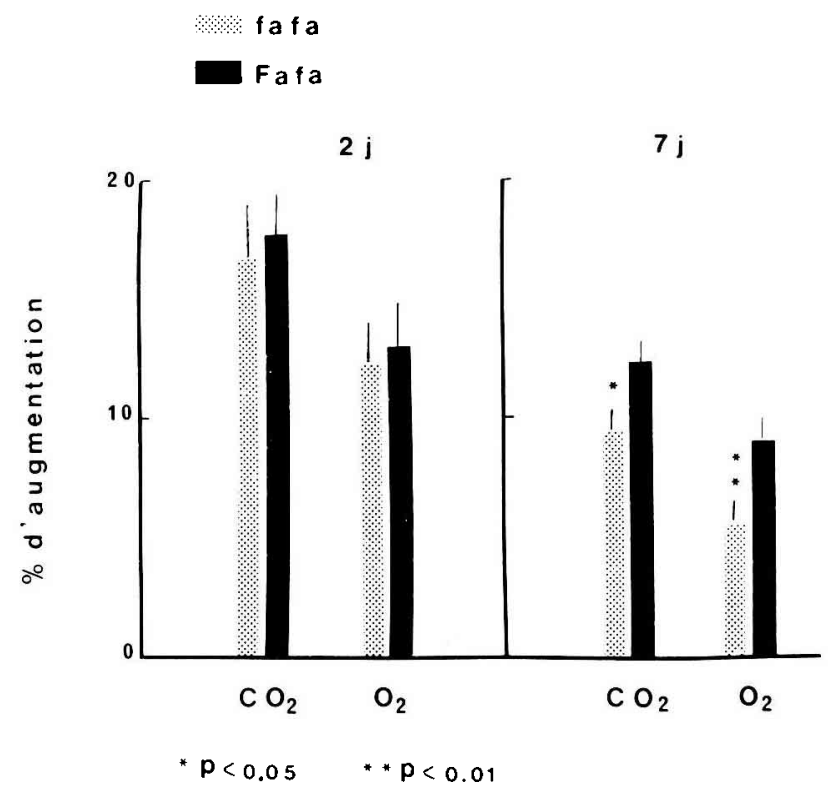

FIG. 1. - Augmentation des échanges gazeux après réalimentation.

L'effet de la réalimentation sur le niveau des échanges gazeux était exprimé en pourcentage d'augmentation de l'excrétion du $\mathrm{CO}_{2}$ et de la consommation d' $\mathrm{O}_{2}$. Le nombre des animaux était : à 2 jours, $24 \mathrm{fa} / \mathrm{fa}$ et $25 \mathrm{Fa} / \mathrm{fa}$; à 7 jours : $31 \mathrm{fa} / \mathrm{fa}$ et $27 \mathrm{Fa} / \mathrm{fa}$. Les différences statistiquement significatives entre les 2 génotypes sont indiquées par $^{*}: P<0,05$ et $^{* *}: P<0,01$.

Cette différence n'étant pas liée à une différence dans le niveau des ingesta entre les 2 génotypes (tabl. 1), nos résultats montrent donc qu'un déficit de la thermogenèse induite par l'alimentation, liée probablement à une anomalie de la capacité thermogénétique du tissu adipeux brun (Bazin et al., 1984), existe chez le 
fa/fa à l'âge de 7 jours. Cependant la différence dans l'augmentation des dépenses énergétiques liée à ce type de thermogenèse entre les 2 génotypes à 7 jours, bien que significative, est faible $(3 \%)$ et ne peut être mise en évidence que dans des conditions artificielles de "jeûne » et de réalimentation, contrairement à l'importance différence (20 à $30 \%$ ) dans l'augmentation de la thermogenèse induite par le froid qui était observée dans les conditions habituelles de température ambiante des ratons (Planche et Joliff, 1985).

En outre, contrairement aux anomalies de la réponse au froid (Planche et Joliff, 1985) et du métabolisme oxydatif du tissu adipeux brun (Bazin et al., 1984) le déficit de réponse thermogénique à l'alimentation est absent chez le $\mathrm{fa} / \mathrm{fa}$ à l'âge de 2 jours. II ne semble donc pas être un facteur primaire mais plutôt une conséquence du développement de l'obésité déjà présente chez le fa/fa à l'âge de 7 jours (Boulangé et al., 1979 ; Planche et al., 1983), I'hypertrophie et I'hyperactivité du tissu adipeux blanc favorisant l'utilisation des nutriments pour la synthèse des lipides de réserve au détriment de leur oxydation notamment au niveau du tissu adipeux brun.

11e Réunion du groupe Développement I.N.R.A., Montpellier, 22-24 mai 1985.

\section{References}

BAZIN R., ETEVE D., LAVAU M., 1984. Evidence for decreased G.D.P. binding to brown adipose tissue mitochondria of obese Zucker $(\mathrm{fa} / \mathrm{fa})$ rats in the very first days of life. Biochem. J. 221, 241-245.

BOULANGÉ A., PLANCHE E., DE GASQUET P., 1979. Onset of genetic obesity in the absence of hyperphagia during the first week of life in the Zucker rat (fa/fa). J. Lipid Res., 20. 857-864.

CONKLIN P., HEGGENESS W., 1971. Maturation of temperature homeostasis in the rat. $\mathrm{Am} . \mathrm{J}$. Physiol., 220, 333-336.

FOSTER D. O., FRYDMAN L., 1979. Tissue distribution of cold induced-thermogenesis in conscious warm or cold-acclimated rats reevaluated from changes in tissue blood flow: The dominant role of brown adipose tissue in the replacement of shivering by non shivering thermogenesis. Can. J. Physiol. Parmacol, 57, 257-270.

GODBOLE V. Y., GRUNDLEGER M. L., PASQUINE T. A., THENEN S. W., 1981. Composition of rat milk from day 5 to 20 of lactation and milk intake of lean and preobese Zucker pups. $J$. Nutr., 111, 480-487.

GODBOLE V. Y., YORK D. A., BLOXHAM P., 1978. Developmental changes in fatty (fa/fa) rats : Evidence for defective thermogenesis preceding the hyperlipogenesis and hyperinsulinemia. Diabetologia, 15, 41-44.

HOLT S., YORK D. A., FITSIMMONS J. T. R., 1983. The effects of corticosterone, cold exposure and overfeeding with sucrose on brown adipose tissue of obese Zucker rats (fa/fa). Biochem. J., 214, 215-223.

KAPLAN L. M., 1979. Consumption of 02 and early detection of fa/fa genotype in rats. Metabolism, 28, 1147-1151.

PLANCHE E., JOLIFF M., 1985. Evolution des dépenses énergétiques du rat Zucker au cours de la première semaine de la vie. Reprod. Nutr. Dévelop., 25, 321-327.

PLANCHE E., JOLIFF M., de GASQUET P., Le LIEPVRE X., 1983. Evidence of a defect in energy expenditure in 7-day old Zucker rat $(\mathrm{fa} / \mathrm{fa})$. Am. J. Physiol., (Endocrinol. Metab.), 245, E107E113.

ROTHWELL N. Y., SAVILLE M. E., STOCK M. J., 1981. Acute effects of food, 2-deoxy-D glucose and noradrenaline on metabolic rate and brown adipose tissue in normal and atropinised lean and obese (fa/fa) Zucker rats. Pfluegers Arch., 392, 172-177. 
SCHMIDT I., KAUL R., CARLISLE H. J., 1984. Body temperature of huddling newborn Zucker rats. Pfluegers Arch., 401, 418-420.

SNEDECOR G. W., COCHRAN W. G., 1967. Statistical methods, 472-477. 6th ed. lowa State Univ. Press, Ames IA.

STERN J. S., INOKUCHI T., CASTONGUAY T. W., WICKLER S. J., HORWITZ B. A., 1984. Scapular brown fat removal enhances development of adiposity in cold-exposed obese Zucker rat. Am. J. Physiol., 247 (regulatory integrative comp. Physiol. 16), R918-R926.

TRAYHURN P., THURLBY P. L., JAMES W. P. T., 1976. A defective response to cold in the obese $(\mathrm{ob} / \mathrm{ob}$ ) mouse and the obese Zucker (fa/fa) rat. (Abstr.). Proc. Nutr. Soc., 35, 133A.

TRIANDAFILLOU J., HIMMS-HAGEN J., 1983. Brown adipose tissue in genetically obese $(\mathrm{fa} / \mathrm{fa})$ rats : response to cold and diet. Am. J. Physiol., 244 (Endocrinol. Metab.), 7, E145-E150. 